\title{
AVALIAÇÃO DA TÉCNICA DE DENERVAÇÃO PERCUTÂNEA DA ARTICULAÇÃO COXOFEMORAL NO TRATAMENTO DA DISPLASIA EM CÃES
}

Recebido: $17 / 09 / 2016$

HYPPOLITO, William Cordeiro ${ }^{1}$; ALVES, José Edgard de Oliveira ${ }^{2}$; RAMOS, Renato Moran ${ }^{3}$; Aceito: 08/04/2017 OLIVEIRA, André Lacerda Abreu de ${ }^{4}$.

${ }^{1}$ Residente de Cirurgia de Pequenos Animais da Universidade Estadual do Norte Fluminense; ${ }^{2}$ Residente de Anestesiologia Veterinária da Universidade Estadual do Norte Fluminense; ${ }^{3}$ Professor da Universidade Iguaçu; ${ }^{4}$ Professor da Universidade Estadual do Norte Fluminense.

\section{RESUMO}

A displasia coxofemoral é uma afecção da articulação coxofemoral que acomete mais comumente cães de raças grandes. Várias técnicas cirúrgicas têm sido utilizadas para o tratamento dessa afecção e, dentre elas, se destaca a denervação percutânea da articulação coxofemoral em função de seu rápido tempo de recuperação, por ser pouco agressiva e por requerer um menor período anestésico. O objetivo do presente estudo foi avaliar a eficácia da técnica de denervação da articulação coxofemoral no tratamento de cães displásicos. Para tanto, foram utilizados seis cães com massas corporais entre 32 e 41 $\mathrm{kg}$, das raças Dobermann, Pastor Alemão e Rottweiler, diagnosticados com displasia coxofemoral e submetidos a cirurgia de denervação percutânea da articulação coxofemoral. Os resultados mostraram que a técnica de denervação da articulação coxofemoral apresentou resultados favoráveis e satisfatórios para o tratamento da displasia coxofemoral em caninos.

Palavras-chave: Ortopedia. Artropatia. Clínica cirúrgica. 


\section{INTRODUÇÃO}

A displasia coxofemoral é uma afeç̧ão da articulação coxofemoral que acomete principalmente cães de raças grandes, especialmente Pastores Alemães, Setters Ingleses, Cães dos Pirineus e São Bernardos (GEROSA, 1995). Ela é caracterizada por uma série de alterações na articulação que incluem articulação frouxa, cavidades acetabulares superficiais, tumefação, subluxação, frouxidão ou rompimento do ligamento redondo do fêmur, achatamento da cabeça femoral e produção de osteófitos periarticulares (ETTINGER; FELDMAN, 1997). No entanto, fatores nutricionais, genéticos e ambientais também estão ligados a essa afecção, além de haver associação também com fatores anormais na musculatura esquelética da pelve. Atualmente aceita-se que a causa da displasia coxofemoral é uma fraqueza da musculatura pélvica devido a fatores hereditários, sendo que, desta forma, não há força suficiente para manter a cabeça do fêmur no acetábulo, resultando no desgaste articular (CARDINET et al., 1997).

O tratamento para a displasia coxofemoral pode ser conservativo ou cirúrgico. O tratamento conservativo constitui em ajustes nutricionais, diminuição do peso do animal, fisioterapia, diminuição da quantidade de exercícios e controle da dor (TOMLINSON; McLAUGHLIN, 1996). Várias técnicas têm sido utilizadas no tratamento cirúrgico da displasia coxofemoral em cães, entre elas a amputação da cabeça do fêmur, que é uma técnica cruenta e que consiste em excisar a cabeça femoral, evitando assim o contato da mesma com o acetábulo e gerando uma pseudo-articulação fibrosa; a substituição total da articulação do quadril, que é um método de custo elevado e de alto risco; e a sinfisiodese púbica e a osteotomia pélvica, que têm seu uso restrito a cães jovens (a primeira só é indicada em cães com menos de 20 semanas de vida). Nesse contexto, aparece também a denervação da articulação coxofemoral como método alternativo a esses tratamentos. Esta técnica baseia-se na raspagem do periósteo da borda acetabular com o intuito de eliminar a inervação sensitiva da articulação do quadril e gerar analgesia para o animal (KINZEL et al., 1998).

O presente artigo teve como objetivo relatar o estado clínico dos pacientes antes e após a cirurgia de denervação percutânea da articulação coxofemoral, assim como avaliar as vantagens, desvantagens, possíveis complicações e resultados desta técnica cirúrgica. 


\section{MATERIAL E MÉTODOS}

O presente estudo foi realizado no Hospital Veterinário da Universidade Estadual do Norte Fluminense Darcy Ribeiro (UENF), utilizando seis cães com massas corporais entre 32 e 41 $\mathrm{kg}$, das raças Dobermann, Pastor Alemão e Rottweiler, com idades entre 5 e 12 anos, sendo 4 fêmeas e 2 machos previamente diagnosticados com displasia coxofemoral.

Para realização do procedimento, os pacientes foram submetidos à anestesia geral. Para tal, foram premedicados com acepromazina ${ }^{1}$ na dose de $0,05 \mathrm{mg} \mathrm{kg}^{-1}$ e morfina ${ }^{2}$ na dose de 0,5 mg. $\mathrm{kg}^{-1}$, ambos por via intramuscular. A indução anestésica foi realizada com $5 \mathrm{mg} \cdot \mathrm{kg}^{-1} \mathrm{de}$ propofol $^{3}$ por via intravenosa e manutenção com isoflurano ${ }^{4}$ em oxigênio a $100 \%$ em circuito semi-fechado.

Os pacientes foram posicionados em decúbito lateral com a articulação a ser operada voltada para cima. Foram realizadas palpações da crista ilíaca, do trocânter maior e da tuberosidade isquiática que serviram de referência para o eixo longitudinal da hemipelve e do corpo do ílio. Aproximadamente um centímetro craniodorsal ao trocânter maior foi realizada uma incisão cutânea de $5 \mathrm{~mm}$ com lâmina de bisturi no 24, pela qual foi introduzido, a campo fechado, perpendicularmente à pele e à superfície do corpo do ílio, um pino de Steinmann de 3,5 mm de diâmetro, por meio dos ventres musculares glúteos, até atingir-se a superfície óssea. Posteriormente, foram realizados movimentos repetidos de raspagem do periósteo em sentido semicircular, nas faces cranial, craniodorsal e dorsal da borda acetabular, tomando-se o cuidado de evitar a perda de contato da ponta do pino intramedular com a superfície óssea nas faces dorsal e ventral do corpo ilíaco.

No pós-operatório imediato, os animais foram medicados com cetoprofeno ${ }^{5}$ na dose de 1 mg.kg-1 por via subcutânea e, após a alta hospitalar, foi administrada a suspensão oral ${ }^{6}$ deste mesmo AINE por mais 4 dias, respeitando-se um intervalo de 24 horas entre as

\footnotetext{
${ }^{1}$ Acepran ${ }^{\circledR} 0,2 \%$, Vetnil, Louveira, SP, Brasil.

${ }^{2}$ Dimorf $^{\circledR} 1 \%$, Cristália, Itapira, SP, Brasil.

${ }^{3}$ Fresofol $^{\circledR} 1 \%$, Fresenius Kabi, Barueri, SP, Brasil.

${ }^{4}$ Isoforine, Cristália, Itapira, SP, Brasil.

${ }^{5}$ Ketofen ${ }^{\circledR} 1 \%$, Merial. Campinas, SP, Brasil.

${ }^{6}$ Profenid $^{\circledR}$, Sanofi, São Paulo, SP, Brasil.
} 
administrações. Os animais foram avaliados diariamente durante os 7 primeiros dias após a realização da denervação e, posteriormente, avaliados no $14^{\circ}$ dia após a cirurgia e, finalmente, 18 meses após o procedimento.

\section{RESULTADOS E DISCUSSÃO}

Dos seis animais estudados, três apresentavam atrofia na musculatura de ambos os membros pélvicos e um deles apenas no membro pélvico esquerdo, ao passo que os outros dois animais não apresentavam atrofia da musculatura em nenhum dos membros pélvicos, apesar de se observar a claudicação nestes animais. Estes animais apresentavam grau avançado de displasia coxofemoral na avaliação radiográfica (figura 1), onde observava-se contorno irregular da cabeça do fêmur e uma intensa reação periosteal no acetábulo, sendo estes achados comuns em casos de displasias severas e crônicas.

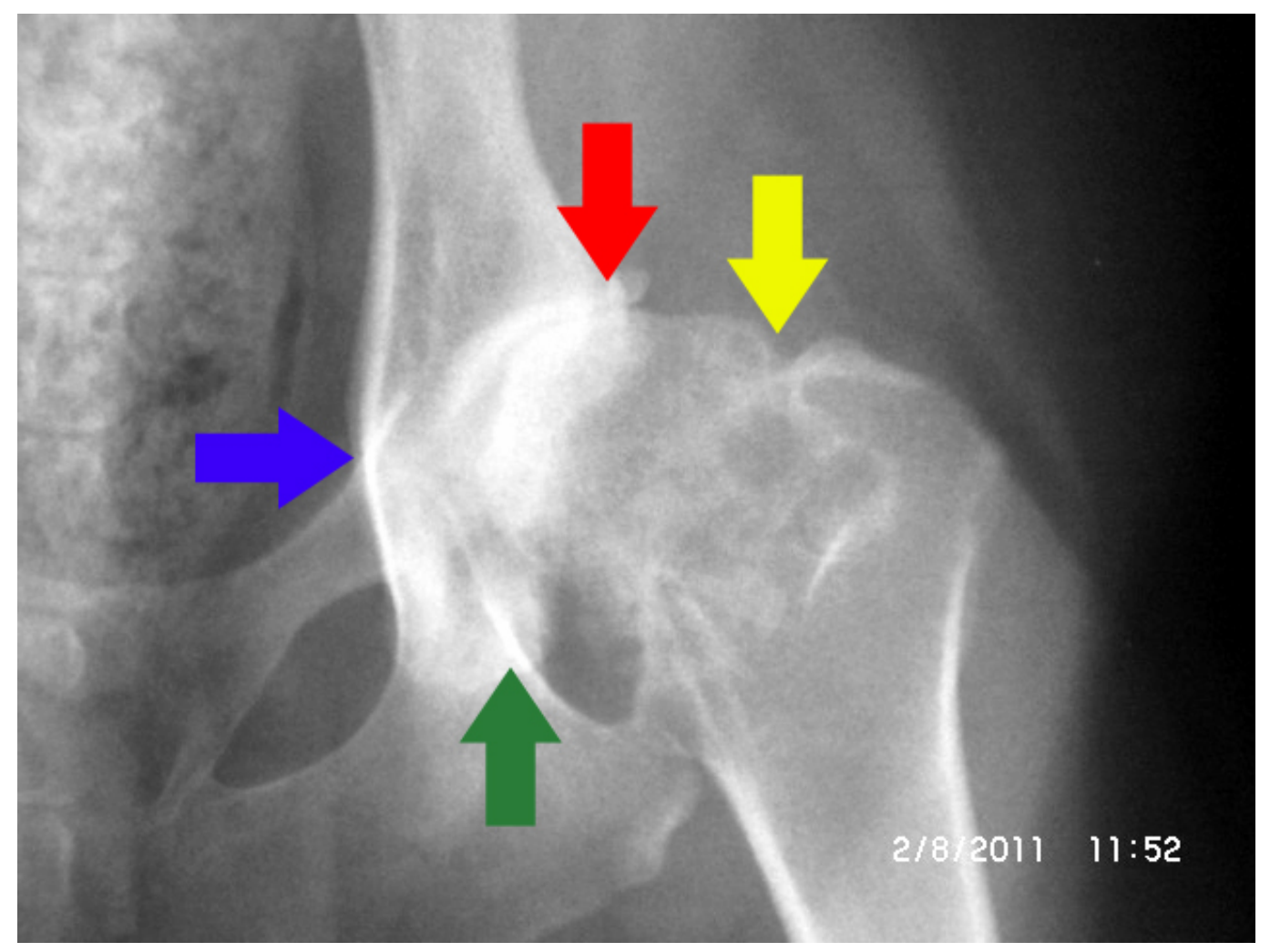

Figura 1 - Radiografia ventro-dorsal da articulação coxofemoral esquerda de uma fêmea da raça Doberman de sete anos de idade e $38 \mathrm{~kg}$, submetida posteriormente ao procedimento de denervação da articulação coxofemoral. Evidencia-se arrasamento acetabular (flecha verde), espessamento do colo femoral (flecha amarela), deformidade da cabeça do fêmur (flecha vermelha) e reação periosteal do acetábulo (flecha azul). 
O procedimento pôde ser realizado rapidamente, com um tempo operatório de aproximadamente 20 minutos, sendo que, em média, foram gastos cerca de 10 minutos para a denervação de cada articulação em cada animal.

Em todos os animais o pino foi introduzido através de uma incisão cutânea de aproximadamente $5 \mathrm{~mm}$. Em nenhum dos animais houve a necessidade de se realizar a sutura da incisão ao término do procedimento, em função de suas dimensões reduzidas.

Dos seis animais estudados no presente trabalho, um apresentou melhora do quadro clínico (melhora na marcha) no pós-operatório imediato, um obteve melhora clínica no $4^{\circ}$ dia de pós-operatório, e os quatro animais restantes apresentaram melhora clínica no $5^{\circ}$ dia de pós-operatório. $\mathrm{Na}$ entrevista realizada com os proprietários no $14^{\circ}$ dia após o procedimento, não foi relatada recidiva do quadro clínico em nenhum dos animais. Após 18 meses todos os animais estudados apresentavam-se clinicamente bem, sem sinais de claudicação ou dor decorrentes da displasia coxofemoral.

Os resultados aqui apresentados estão de acordo com aqueles descritos na literatura, a exemplo de Selmi et al. (2009) que encontraram resultados favoráveis utilizando a mesma técnica. Ferrigno et al. (2004), observaram melhora clínica na primeira semana de pósoperatório, resultados que também concordam com os apresentados no presente trabalho.

Ferrigno et al. (2007) encontraram resultados favoráveis ao realizar a técnica aberta para a denervação da articulação coxofemoral, entretanto, necessitaram de maior tempo cirúrgico e de uma maior quantidade de medicamentos no pós-operatório, sendo necessária a utilização de antibióticos, fato que não foi necessário nos casos estudados neste artigo em função do menor tempo cirúrgico e da utilização de uma técnica pouco invasiva. Outra técnica que demonstra ter um alto grau de sucesso no tratamento cirúrgico da displasia coxofemoral em caninos é a substituição das partes articulares da articulação coxofemoral por próteses metálicas. Olmstead (1995) relatou a eficácia desse tratamento, com remissão total da dor e retorno rápido às atividades normais em $95 \%$ dos casos. No entanto é uma técnica laboriosa, de difícil execução, elevado tempo cirúrgico e alto custo, características não observadas na técnica de denervação percutânea que utilizamos em nosso estudo. 
No tocante ao quadro clínico, um dos principais relatos da literatura com relação às anormalidades físicas encontradas em cães com displasia coxofemoral é a atrofia muscular dos membros pélvicos (HULSE; JOHNSON, 2002), que ocorre por conta da claudicação apresentada pelos animais. Nos casos estudados, quatro dos seis animais apresentavam atrofia muscular em um ou ambos os membros pélvicos, corroborando a literatura consultada, que cita a atrofia muscular como achado comum.

Com relação às raças que apresentaram a displasia da articulação coxofemoral, foram encontrados quatro cães da raça Pastor Alemão, correspondendo com as afirmações de Gerosa (1995), que citou esta raça entre as predispostas à doença e ainda relatou que a maioria dos animais acometidos eram de raças grandes, o que também está de acordo com nossos achados.

A cápsula da articulação coxofemoral em cães é inervada na sua porção crânio-lateral por ramos do nervo glúteo cranial, na porção crânio-medial por ramos do nervo femoral e na porção caudo-medial por ramos do nervo obturatório, que percorrem inicialmente o periósteo do corpo do ílio antes de atingir a cápsula articular (ROCHA et al., 2013). Cabe relatar que em nenhum dos procedimentos houve lesão do nervo ciático ou do nervo glúteo cranial, tampouco da artéria e veia glútea. Isto deve-se provavelmente ao fato de que a região abordada é cranial à região das estruturas citadas, diminuindo, assim, as chances de que houvesse qualquer contato entre o pino de Steinmann e as estruturas supracitadas.

Nenhum dos animais apresentou piora no quadro clínico nos primeiros dias após a cirurgia. Um dos animais, inclusive, obteve melhora no quadro clínico no pós-operatório imediato. Estas informações contradizem os resultados descritos por Selmi et al. (2009). Nossos resultados também contradizem àqueles encontrados por Nogueira et al. (2005), que relataram que apenas $60 \%$ dos animais apresentaram melhora durantes os primeiros sete dias de pós-operatório, o que foi observado em maior percentual no presente artigo. Todos os animais submetidos ao tratamento da displasia coxofemoral através da técnica de denervação percutânea apresentaram melhora clínica, o que representou um total de $100 \%$ do universo estudado. Este resultado é um pouco melhor do que aqueles encontrados por Kinzel et al. (2002), Braun et al. (2003) e Ferrigno et al. (2004), provavelmente em função do 
reduzido número de animais utilizados neste estudo em comparação com os demais experimentos supracitados. Além disso, os cães selecionados para a realização desta técnica apresentavam sinais clínicos compatíveis com dor na articulação coxofemoral, manifestados através da claudicação e consequente atrofia muscular por conta da subutilização da musculatura pélvica, com isso, a eliminação da inervação sensitiva da articulação contribuiu diretamente para o alívio do quadro álgico e o elevado grau de êxito neste estudo.

\section{CONCLUSÃO}

Conclui-se que a denervação percutânea da articulação coxofemoral é uma técnica rápida, eficaz e pouco onerosa para o tratamento da displasia coxofemoral, uma vez que a mesma apresentou resultados favoráveis em curto espaço de tempo, contribuindo, assim, para uma melhora na qualidade de vida dos cães portadores da doença.

\section{ASSESSMENT OF THE HIP JOINT PERCUTANEOUS DENERVATION TECHNIQUE IN THE TREATMENT OF HIP DYSPLASIA IN DOGS}

\section{ABSTRACT}

$\mathrm{T}$ he hip dysplasia is a disease that affects the hip joint and occurs more commonly in dogs of big breeds. Several surgical techniques have been used to treat this disorder and, amongst them, stands out the percutaneous denervation of the hip joint due to its fast recovery time, the patient remains a minor period under anesthesia and it is less aggressive This study aims evaluate the efficacy of the hip joint percutaneous denervation technique in treatment of dogs with hip dysplasia. Six Dobermann, German Shepherd and Rottweiler dogs weighing between 32 and $41 \mathrm{~kg}$, diagnosed with hip dysplasia and submitted to percutaneous denervation of hip joint surgery were used. Our results proved that the denervation of the hip joint technique presents favorable and satisfactory results for the treatment of hip dysplasia in dogs.

Keywords: Orthopedics. Arthropathy. Clinical surgery. 


\section{EVALUACIÓN DE LA TÉCNICA DE DENERVACIÓN PERCUTÁNEA DE LA ARTICULACIÓN COXOFEMORAL EN EL TRATAMIENTO DE LA DISPLASIA EN PERROS}

\section{RESUMEN}

E objetivo de este estudio fue evaluar la eficacia de la técnica de denervación percutánea de la articulación coxofemoral en el tratamiento de perros displásicos. La displasia coxofemoral es un desorden de la articulación coxofemoral que se produce con mayor frecuencia en perros de razas grandes. Diversas técnicas quirúrgicas han sido utilizadas para tratar esta enfermedad y entre ellas se encuentra la denervación percutánea de la articulación coxofemoral, debido a su rápido tiempo de recuperación, a ser poco agresiva y a la necesidad de un período anestésico menor. Fueron utilizados seis perros con pesos entre 32 y $41 \mathrm{~kg}$, de las razas Doberman, Pastor Alemán y Rottweiler, previamente diagnosticados con displasia coxofemoral, y que fueron sometidos a cirugía de denervación percutánea de la articulación coxofemoral. Nuestros resultados muestran que la técnica de denervación de la articulación coxofemoral presenta resultados favorables y satisfactorios para el tratamiento de la displasia coxofemoral en los perros.

Palabras clave: Ortopadía. Artropatía. Clínica quirúrgica.

\section{REFERÊNCIAS}

BRAUN, D.; LAUTERSACK, O.; SCHIIMKE, E. Dorsale denervation der hüftgelenkskapsel beim hund ergebnisse einer langzeitstudie. Kleintierpraxis, v. 4, p. 185-244, 2003.

CARDINET, G. H.; KASS, P. H.; WALLACE, L. J.; et al. Association between pelvic muscle mass and canine hip dysplasia. Journal of the American Veterinary Medical Association, v. 210, n. 10, p. 1466-1473, 1997.

ETTINGER, S. J.; FELDMAN, E. C. Tratado de Medicina Interna Veterinária. São Paulo: Manole, 1997.

FERRIGNO, C. R. A.; D'ÁVILA, R. S.; YAMAMOTO, E. Y.; et al. Estudo da técnica de denervação da cápsula articular coxofemoral no tratamento da dor em cães com displasia coxofemoral: resultados preliminares. Brazilian Journal of Veterinary Research and Animal Science, v. 41, p. 169-170, 2004.

FERRIGNO, C. R. A.; SCHMAEDECKE, A.; OLIVEIRA, L. M.; et al. Denervação acetabular cranial e dorsal no tratamento da displasia coxofemoral em cães: 360 dias de evolução de 97 casos. Pesquisa Veterinária Brasileira, v. 27, p. 333-340, 2007. 
GEROSA, R. M. La displasia de la cadera desde una visión biomecánica. Revista de Medicina Veterinaria, v. 76, p. 69-71, 1995.

HULSE, D. A.; JOHNSON, A. L. Tratamento da Doença Articular. In: FOSSUM, T. W. Cirurgia de Pequenos Animais. 2. ed. São Paulo: Roca, 2002. P. 1042-1087.

KINZEL, S.; FASSELT, R.; PRESCHER, A.; et al. Sensory innervation of the hip joint capsule in dogs. Tierarztliche Praxis, v. 26, n. 5, p. 330-335, 1998.

KINZEL, S.; HEIN, S.; VON SCHEVEN, C.; et al. 10 years experience with denervation of the hip joint capsule in the treatment of canine hip joint dysplasia and arthrosis. Berliner und Münchener Tierärztliche Wochenschrift, v. 115, p. 53-56, 2002.

NOGUEIRA, S. R.; ROCHA, L. B.; TUDURY, E. A. Utilização do índice de distração no diagnóstico da displasia coxofemoral canina. Clínica Veterinária, v. 10, n. 54, p. 28-41, 2005.

OLMSTEAD, M. L. Canine cemented total hip replacements: state of the art. Journal of Small Animal Practice, v. 36, n. 9, p. 395-399, 1995.

ROCHA, L. B.; TUDURY, E. A.; ROEHSIG, C.; BARAÚNA, D.; CHIORATTO, R.; ARAÚJO, F. P.; KEMPER, B. Denervação articular coxofemoral em cães com doença articular degenerativa secundária à displasia. Ciência Animal Brasileira, v. 14, n. 1, p. 120-134, 2013.

SELMI, A. L.; PENTEADO, B. M.; LINS, B. T. Denervação capsular percutânea no tratamento da displasia coxofemoral canina. Ciência Rural, v. 39, n. 2, p. 460-466, 2009.

TOMLINSON, J.; McLAUGHLIN, R. Medically managing canine hip dysplasia. Veterinary Medicine, v. 91, n. 1, p. 48-53, 1996. 